\title{
Improving FAIMS Sensitivity Using a Planar Geometry with Slit Interfaces
}

\author{
Ridha Mabrouki, Ryan T. Kelly, David C. Prior, \\ Alexandre A. Shvartsburg, Keqi Tang, and Richard D. Smith \\ Biological Sciences Division, Pacific Northwest National Laboratory, Richland, Washington, USA
}

\begin{abstract}
Differential mobility spectrometry or field asymmetric waveform ion mobility spectrometry (FAIMS) is gaining broad acceptance for analyses of gas-phase ions, especially in conjunction with largely orthogonal separation methods such as mass spectrometry (MS) and/or conventional (drift tube) ion mobility spectrometry. In FAIMS, ions are filtered while passing through a gap between two electrodes that may have planar or curved (in particular, cylindrical) geometry. Despite substantial inherent advantages of the planar configuration and its near-universal adoption in current stand-alone FAIMS devices, commercial FAIMS/MS systems have employed curved FAIMS geometries that can be more effectively interfaced to MS. Here we report a new planar (p-) FAIMS design with slit-shaped entrance and exit apertures that substantially increase ion transmission in and out of the analyzer. The entrance slit interface effectively couples p-FAIMS to multi-emitter electrospray ionization (ESI) sources, improving greatly the ion current introduced to the device and allowing liquid flow rates up to $\sim 50 \mu \mathrm{L} / \mathrm{min}$. The exit slit interface increases the transmission of ribbon-shaped ion beams output by the p-FAIMS to downstream stages such as a MS. Overall, the ion signal in ESI/FAIMS/MS analyses increases by over an order of magnitude without affecting FAIMS resolution. (J Am Soc Mass Spectrom 2009, 20, 1768-1774) @ 2009 American Society for Mass Spectrometry
\end{abstract}

$\mathrm{F}$ ield asymmetric waveform ion mobility spectrometry (FAIMS) is increasingly recognized as a powerful separation technique that can process ions in gases in milliseconds at ambient conditions [1-6] and is readily combined with other common analytical tools, including liquid chromatography (LC) [7-9] and/or mass spectrometry (MS) [9-13]. The operation of FAIMS has been understood fundamentally $[2,5,14]$ and modeled mathematically and in simulations [10, 15-17]. Briefly, the mobility of an ion in a gas mainly depends on $E / N$, where $E$ is the electric field intensity and $N$ is the gas number density [18]:

$$
K(E / N)=K(0)[1+f(E / N)]
$$

where $K(0)$ is the mobility in zero-field limit and $f(E / N)$ is a function of field strength. This allows sorting ions by the difference of their mobility at high and low $E / N$ [that is, the form of $f(E / N)$ ], regardless of $K(0)$. On the contrary, the conventional drift tube ion mobility spectrometry (DTIMS) separates ions by absolute mobility, and the field is commonly weak enough to equate $K(E / N)$ to $K(0)$. In FAIMS, ions experience alternating strong and weak fields of opposite polarity, generated by a periodic asymmetric waveform [1] applied in the gap between two electrodes in the direction perpendic-

Address reprint requests to Dr. R. D. Smith, Biological Systems Analysis and Mass Spectrometry, Pacific Northwest National Laboratory, 3335 Q Ave. (K8-98), P.O. Box 999, Richland, WA 99352, USA. E-mail: rds@pnl.gov ular to a gas flow. Ions carried through FAIMS by the flow are displaced toward one of the electrodes in each waveform cycle and would eventually be lost. To prevent this for species with a specific mobility difference between high and low $E / N$ and keep them stable in the gap, the net displacement induced by the waveform is offset by adding to it an appropriate dc potential (the compensation voltage, CV) $[2,14]$. Then, each ion species passes FAIMS at a CV that is unique for a given waveform peak amplitude (the dispersion voltage, DV). The proper value for any ion can be found by scanning $\mathrm{CV}$ over a broad range while monitoring the ions emerging from FAIMS by some detector, for example a charge collector or a following MS stage. For easier data comparison across different FAIMS geometries, $\mathrm{CV}$ is converted to the compensation field, $E_{\mathrm{C}}$, by dividing over the gap width.

The FAIMS separation by mean derivative of $K(E / N)$ function is largely independent of methods employing other physical principles such as DTIMS or LC based on adsorption properties. Hence integrating FAIMS with LC and/or DTIMS significantly increases selectivity and helps isolating and identifying trace components in complex samples. In particular, inserting FAIMS into the LC/ESI/MS pipeline raises the separation peak capacity, improving the spectral quality and removing isobaric interferences $[8,9,19-24]$.

All commercial ESI/FAIMS/MS platforms employ coaxial cylindrical FAIMS electrodes, where ions tra- 
verse the annular gap either parallel to the axis [25, 26] or (in the "side-by-side" arrangement) along the circumference [27]. In either case, inhomogeneous electric field in curved gaps focuses ions to the median, which enhances ion transmission through FAIMS. However, focusing limits the resolving power $R$ by rendering species with differing CVs stable at different radial positions in the gap and, by calculations [28], the optimum resolution/sensitivity balance at any $R$ is provided by planar FAIMS. Further, the focusing strength is controlled by the shape of $K(E / N)$, causing discrimination in FAIMS against poorly focusing species that complicates quantification and compresses the dynamic range [16]. Species that are focused with one waveform polarity [also decided by the form of $K(E / N)$ ] are defocused with the opposite polarity. Covering both polarities means duplication of analyses, which halves the experimental duty cycle. The homogeneous field in planar FAIMS does not focus ions, and all species can be analyzed simultaneously without discrimination by focusing properties. Those advantages are widely recognized and most stand-alone FAIMS analyzers have employed the planar (p-) geometry [29-32]. Though p-FAIMS/MS coupling has been demonstrated, its broad adoption has been impeded by poor sensitivity ensuing from unrestrained ion beam expansion in the gap and large losses at the interface. This problem can be addressed by replacing the standard circular orifice at the MS inlet by a slit aperture that better captures ribbon-like beams exiting p-FAIMS, [28], followed by electrodynamic ion funnel for their re-focusing into tight circular beams for MS analysis [33]. Here we explore this solution in depth, looking at the merits of various slit geometries.

Another path to more sensitive ESI/FAIMS/MS analyses is increasing the ion flux generated by ESI and its transmission into the FAIMS stage. The ionization efficiency of ESI is boosted by reducing the liquid flow rate (Q) to yield smaller, more readily desolvated charged droplets [34]. For a fixed flow of infused analyte [35-37] or eluent from LC separation, the reduction can be achieved by partitioning the flow over an array of ESI emitters [35-38]. Multi-ESI sources have previously been coupled to MS using multi-inlet/ion funnel interfaces that can collect ions from a large area [39]. Present ESI/FAIMS interfaces using a curtain plate/orifice with a single circular inlet cannot efficiently sample the ion current from an ESI emitter array because of geometric mismatch and perhaps charge-capacity constraints: a multi-aperture FAIMS inlet is clearly needed.

Penetration of ESI solvent vapors into FAIMS causes large CV shifts that preclude reproducible analyses. The gas counterflow through the curtain plate prevents this penetration and ensures stable CVs at lower $Q$, but the amount of vapors (proportional to $Q$ ) apparently overcomes that counterflow at higher $Q$ and CVs drift, depending on $Q$ [40]. Emitter arrays reduce the production of vapors per unit volume, which should make repelling them from the FAIMS gap easier and, thus, permit greater liquid flows with ESI/FAIMS analyses.

Here we report a p-FAIMS with multi-aperture slit front and back interfaces that enable in-plane processing of ion beams generated by linear ESI emitter arrays through FAIMS and into downstream stages. The ion transmission efficiencies both into and out of p-FAIMS improve significantly, raising the final ion signal by an order of magnitude compared with ESI/FAIMS/MS systems with standard circular interfaces. These FAIMS devices are compatible with ESI liquid flows up to (at least) $50 \mu \mathrm{L} / \mathrm{min}$.

\section{Experimental}

\section{Sample}

Measurements used a $10 \mu \mathrm{M}$ solution of reserpine and a solution of four peptides $[1 \mu \mathrm{M}$ each of bradykinin (Bk), angiotensin I (Ang), fibrinopeptide a (Fib), and neurotensin (Neu)] in 50:49:1 water/methanol (HPLC grade)/acetic acid mixture, with water purified through a Barnstead Nanopure Infinity system (Dubuque, IA).

\section{ESI Sources}

We evaluated ESI arrays comprising 5, 9, and 19 emitters spread over a $10-\mathrm{mm}$ linear segment versus a single emitter benchmark. Thus emitters were spaced by 0.5 $\mathrm{mm}$, sufficing to avoid mutual interference in the ESI operation [41]. Emitters with $20 \mu \mathrm{m}$ i.d. were made by chemical etching of fused-silica capillaries [42], with arrays fabricated as previously described [36]. The ESI voltage (2.9 KV to ground) was generated by a Bertan 205B-03R power supply (Hicksville, NY) and applied to a stainless steel union holding the emitter. The sample was infused by a KD Scientific syringe pump (Holliston, MA) at $Q=0.5-50 \mu \mathrm{L} / \mathrm{min}$, using $0.5 \mu \mathrm{L} / \mathrm{min}$ unless specified otherwise.

\section{New p-FAIMS Design}

The FAIMS unit is a modified high-resolution planar FAIMS developed at the Pacific Northwest National Laboratory (PNNL) [28]. The front interface now features five circular apertures with diameters of $1.60 \mathrm{~mm}$ in the curtain plate and $0.96 \mathrm{~mm}$ in the orifice, equally spaced over superposed segments of $10 \mathrm{~mm}$ to match the length of ESI emitter arrays (Figure 1). Thus, the ratio of curtain plate and orifice aperture areas remains 2:1 as in the single-aperture design [28], keeping the same flow split between the curtain gas for desolvation of ESI droplets (2/3) and carrier gas pulling ions through FAIMS (1/3). A bisinusoidal asymmetric waveform with $750 \mathrm{kHz}$ [28] frequency and DV $=4.0$ $\mathrm{kV}$ was applied to one electrode while the other was connected to a programmable dc power supply for the $\mathrm{CV}$ scan. The gap between the FAIMS exit and the following inlet capillary was fixed at $2 \mathrm{~mm}$ by a 


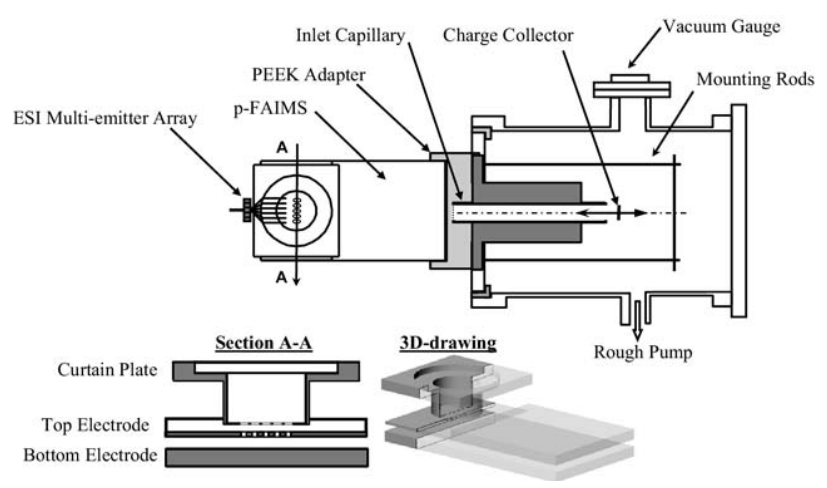

Figure 1. Experimental setup: scheme of p-FAIMS analyzer coupled to a vacuum chamber that contains a charge collector via an inlet capillary (top) and new FAIMS unit with a slit entrance interface (bottom).

polyaryletheretherketone (PEEK) adapter. Experiments used high-purity nitrogen (99.998\%) with the total flow set to $2 \mathrm{~L} / \mathrm{min}$ by mass flow controller (1479A metalsealed mass flow controller; MKS Instruments, Andover, MA). Under these conditions, the protonated reserpine $(\mathrm{m} / \mathrm{z}=609)$ has a distinctive peak [28] at $E_{\mathrm{C}}=$ $-15 \mathrm{~V} / \mathrm{cm}$, where all transmission data were collected.

\section{Inlet Capillary}

The stainless steel inlet capillary comprises an aperture cut in a $0.25 \mathrm{~mm}$ thick plate, followed by a bore of 7.8 $\mathrm{mm}$ diameter and $64 \mathrm{~mm}$ length (Figure 2a). The aperture is a string of 11 equally-spaced circular holes with diameters of $0.127,0.178$, and $0.203 \mathrm{~mm}$ spaced over $4-5 \mathrm{~mm}$, or a rectangular $(4.8 \mathrm{~mm} \times 0.203 \mathrm{~mm})$ slit, termed below designs I, II, III, and IV, respectively. Relative to the cross-section of heated capillary at the MS inlets of our benchmark FAIMS/MS instrument [28] (i.d. of $0.43 \mathrm{~mm}$ and length of $78 \mathrm{~mm}$ ), those apertures have areas of 1 (I), 2 (II), 2.6 (III), and 7 (IV). The conductance of a capillary decreases with increasing length because of viscous friction. The capillary in commercial FAIMS/MS systems (Thermo Fisher, Waltham, MA) is slightly wider (i.d. $=0.5 \mathrm{~mm}$ ) but also longer $(140 \mathrm{~mm})$ than that in our benchmark, and its conductance is close to that of design I. The gap of p-FAIMS matches the orientation of the elongated inlet.

\section{Charge Detector}

In a test platform, ions passing the inlet enter a joined vacuum chamber [34]. Briefly, it is constructed from a 6-in. diameter four-way reducing stainless steel cross and pumped by an E1M18 rough pump (BOC Edwards, Wilmington, MA). The inside pressure ranges from 2 Torr with design I to $\sim 20$ Torr with design IV, as measured by a strain gauge (BOC Edwards). The ion current is collected on a stainless steel disk of $6 \mathrm{~mm}$ diameter, held on a rod and translated along the axis of the inlet capillary to measure the current at various positions. A fraction of the ions exiting the p-FAIMS was not transmitted into the chamber, but lost on the capillary. To allow measuring that fraction, the capillary was electrically insulated from the chamber using a PEEK sleeve. The transmitted $\left(I_{\text {tr }}\right)$ and lost $\left(I_{\text {lost }}\right)$ currents were measured by a picoammeter (model no. 6485; Keithley, Cleveland, $\mathrm{OH}$ ) connected to the charge collector and capillary, respectively, via a BNC vacuum feed through. The current for each setup was acquired three times, in each case averaging 100 consecutive points within the picoammeter.

\section{FAIMS/MS Platform}

Some experiments were performed using FAIMS/MS systems, where ions filtered by FAIMS enter an ion trap MS (Thermo Fisher LCQ) via a standard heated capillary or a triple quadrupole MS (Thermo Fisher TSQ

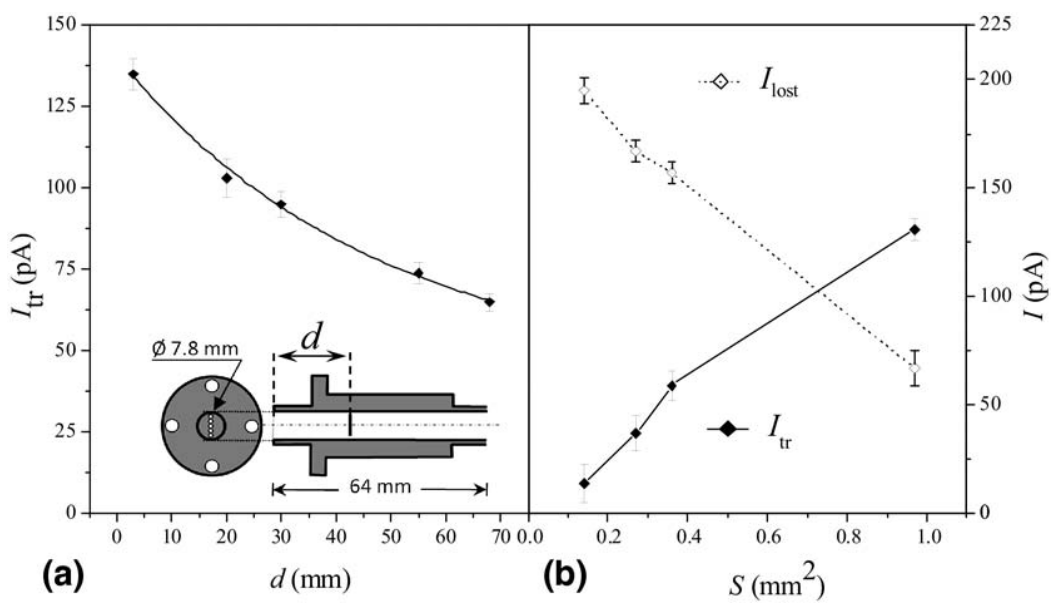

Figure 2. Ion currents incident on the charge collector ("transmitted current", $I_{\text {tr }}$ ) and inlet capillary block ("lost current", $I_{\text {lost }}$ ) depending on (a) the axial position of collector in the capillary and (b) the inlet entrance area. All measurements use five ESI emitters and slit front FAIMS interface, results in (b) include the data for slit inlet designs I-IV (discussed in the text). 
Vantage) via the slit inlet of design IV, followed by tandem ion funnels. Here, no heating is necessary and the capillary was maintained at $70^{\circ} \mathrm{C}$ while the slit aperture was at ambient temperature. The ESI voltage was $3.5 \mathrm{kV}$, the MS inlet was at $35 \mathrm{~V}$ (LCQ) and $\sim 405 \mathrm{~V}$ (TSQ) to ground, and FAIMS was biased at $35 \mathrm{~V}$ with respect to the inlet.

\section{Results and Discussion}

The heated capillary in some ESI/MS interfaces desolvates ions generated by ESI via solvent evaporation due to heating. This process takes a finite time, and prolonging the ion residence in a capillary makes it more complete. However, the expansion of ion beams in a capillary by diffusion and Coulomb repulsion causes significant ion loss to the walls, worsening at longer residence times [34]. The balance of these considerations determines the optimum ion residence time in a capillary and thus its desired length.

In FAIMS/MS, ions are desolvated at the front FAIMS interface and/or by field heating upon injection into the analytical gap [43]. Therefore, a heated capillary at the MS inlet is redundant and, to avoid ion losses inside, can be replaced by a nonheated thin aperture [28]. The benefits of such substitution are characterized here by obtaining $I_{\mathrm{tr}}$ as a function of the charge collector position along the capillary of design IV (with five ESI emitters and slit front FAIMS interface). Moving the collector away from the aperture decreases the current, from $135 \mathrm{pA}$ at the distance of $3 \mathrm{~mm}$ to $65 \mathrm{pA}$ at $66 \mathrm{~mm}$ that is close to the capillary length in our benchmark [28] (Figure 2a). In a regular heated capillary with the i.d. of $\sim 0.5 \mathrm{~mm}$, the loss would likely exceed the present $\sim 50 \%$ because of greater diffusional losses in a narrower passage. So, to emulate FAIMS/MS coupling by an aperture only [28], the current in subsequent studies was measured at $3 \mathrm{~mm}$ distance.

The total ESI current $\left(I_{\text {tr }}+I_{\text {lost }}\right)$ with designs I-IV is consistently $\sim 210 \mathrm{pA}$, but the transmitted portion scales roughly with the aperture area, increasing from $7 \%$ for design I to $\sim 70 \%$ for design IV (Figure $2 \mathrm{~b}$ ). Thus, the open slit in IV captures most ions exiting the present p-FAIMS, versus just $\sim 3 \%$ for the standard circular MS inlet (which is $\sim 40 \%$ that in design I) [28]. Though the pumping limitations of commercial MS systems preclude a $\sim 1 \mathrm{~mm}^{2}$ conductance limit from the atmospheric pressure, that is possible with tandem ion funnel interfaces [44] where the first funnel runs at relatively high-pressure ( $\sim 25$ Torr).

The transmitted current can be raised further by increasing the number of emitters (Figure 3). With the circular ESI/FAIMS interface, changing from 1 to 19 emitters increases $I_{\text {tr }}$ (for the inlet design IV) by just $\sim 25 \%$, from $\sim 75$ to $\sim 93 \mathrm{pA}$. Such modest gain has been expected, as only one emitter can be close enough to the center of single aperture in the curtain plate to contribute much. Changing to a slit interface with a single emitter raises $I_{\text {tr }}$ by a similar amount (Figure 3): simi-

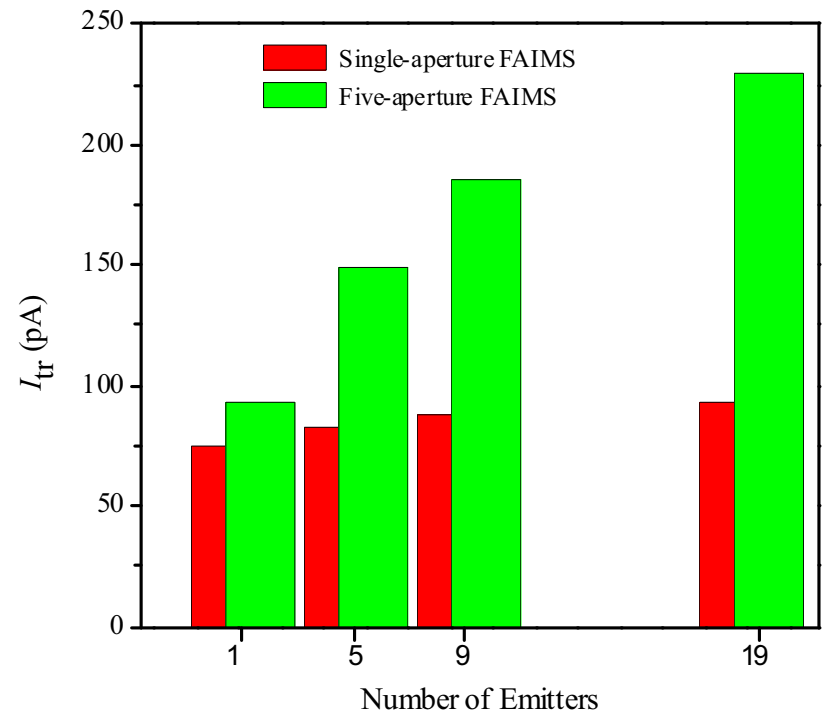

Figure 3. Transmitted ion current with the circular and slit ESI/FAIMS interface, depending on the number of ESI emitters.

larly, all holes except one in the 5-hole slit are too far from the emitter to make much difference. However, combining the 19-emitter array and the slit increases the transmitted current to $\sim 230 \mathrm{pA}$, or three times compared with the benchmark single emitter/circular interface configuration.

The ion current generated by ESI normally scales [45] as $Q^{1 / 2}$. With present system (adopting the design IV), the $I_{\text {tr }}$ values for various ESI emitter array/front FAIMS interface combinations are proportional to $Q^{1 / 2}$ at low $I_{\text {tr }}$ but fall below that trend at higher currents (Figure $4 a)$, suggesting a saturation of the FAIMS ion current capacity. This happens at $I_{\mathrm{tr}} \sim 50 \mathrm{pA}$ for the single emitter/circular interface but $\sim 150-250 \mathrm{pA}$ for the multi-emitter/slit interface configurations, showing that the ion current is limited by the front (ion introduction) FAIMS interface. The rise of saturation current by 3-5 times upon the transition to a slit interface is consistent with the 5-fold increase of aperture area. At any flow rate, $I_{\mathrm{tr}}$ scales as the square root of the number of ESI emitters (Figure $4 \mathrm{~b}$ ). This behavior is a hallmark of multi-emitter ESI in the cone-jet mode [35, 46], confirming that the emitters in present arrays do not unduly obstruct each other's operation.

As the measured ESI/FAIMS spectra exhibit a sharp peak for $\mathrm{H}^{+}$reserpine, the ion current exiting FAIMS at the $\mathrm{CV}$ of peak apex is dominated by those species. Thus, we can estimate the ion utilization efficiency for any emitter configuration, assuming that all detected current reflects reserpine ions. Typical of ESI arrangements $[47,48]$, the ion utilization increases as the liquid flow to the ESI drops (Figure 4c). The efficiency at a given total flow increases for a larger number of ESI emitters, again demonstrating the advantage of multiemitter ESI/FAIMS designs.

A concern is whether increase in ion current upon the use of multi-emitter ESI and/or slit front interface 


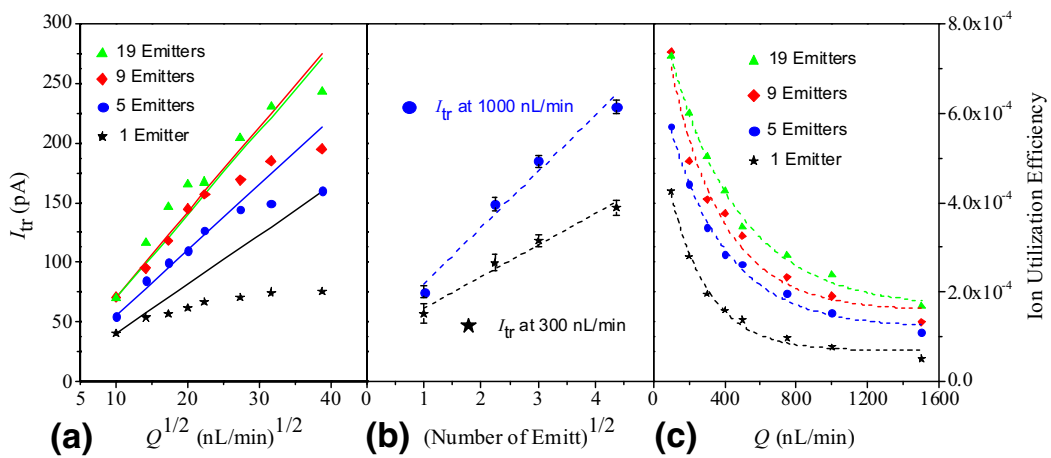

Figure 4. Transmitted ion current for different emitter/front FAIMS interface configurations as a function of the square root of the sample flow rate (a) and number of emitters (b); the ion utilization efficiency depending on the sample flow rate (c).

affects FAIMS resolution. To investigate this, we obtained FAIMS spectra over the range bracketing the reserpine ion peak for all four emitter configurations using the LCQ platform (Figure 5). The normalized spectra in all cases are identical, except for higher baseline in the single-emitter arrangement that is due to lower signal and thus lower signal/noise ratio. This is consistent with simulations showing that peaks in FAIMS do not broaden at higher ion currents [16]. Hence, the ESI emitter arrays and slit interfaces may be used in front of FAIMS without compromising resolution.

To gauge the performance of FAIMS analyzers with slit front interface at higher ESI liquid flows, we increased $Q$ by 100 -fold and analyzed peptide mixtures that were closer to real samples (using the TSQ platform). The solution of Bk (1060 Da), Ang (1296 Da), Fib $(1536 \mathrm{Da})$, and Neu (1672 Da) gave off six major ions (protonated $\mathrm{Bk}^{2+}, \mathrm{Ang}^{2+}, \mathrm{Ang}^{3+}, \mathrm{Fib}^{2+}, \mathrm{Neu}^{2+}$, and $\left.\mathrm{Neu}^{3+}\right)$, Figure 6a. Four of them $\left(\mathrm{Bk}^{2+}, \mathrm{Ang}^{2+}, \mathrm{Ang}^{3+}\right.$,

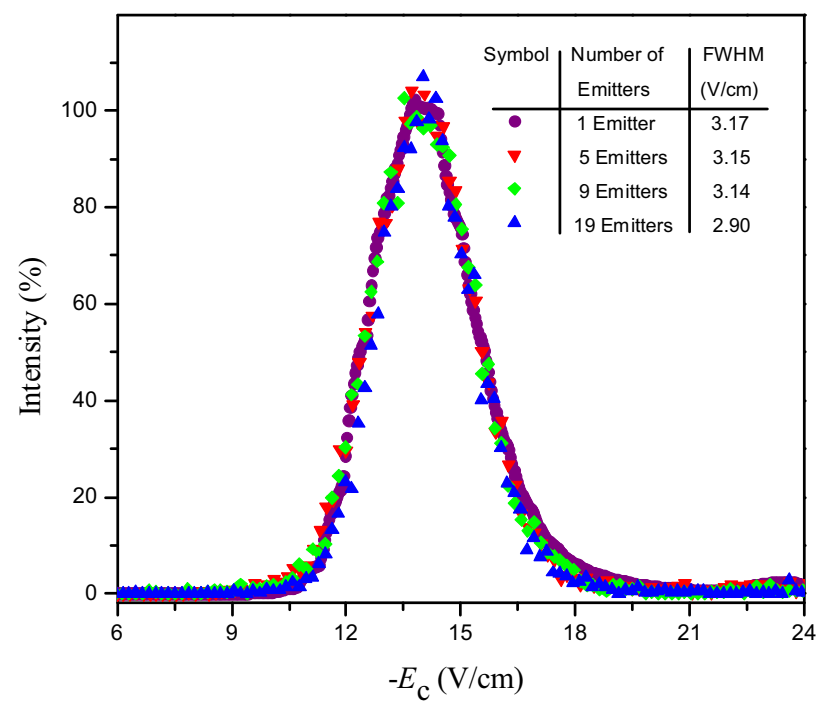

Figure 5. Mass-selected FAIMS spectra for protonated reserpine (obtained at the scan speed of $1 \mathrm{~V} / \mathrm{min}$ ), depending on the number of emitters. The full peak width at half maximum in each case is listed. and $\mathrm{Neu}^{2+}$ ) were fully resolved by FAIMS at $Q=1 \mu \mathrm{L} / \mathrm{min}$, the other two "co-eluting" with some of these four (Figure 6b). The multiple features for $\mathrm{Bk}^{2+}$ and $\mathrm{Fib}^{2+}$ indicated the presence of conformers, in line with previous data [28] for $\mathrm{Bk}^{2+}$. Increasing $Q$ to $50 \mu \mathrm{L} / \mathrm{min}$ did not substantially affect the number or position of features in MS (Figure 6c) or FAIMS (Figure 6d) spectra, though the peaks for two $\mathrm{Fib}^{2+}$ isomers merged. The relative intensities of many features have changed, but this is common upon a 100-fold increase of ESI flow rate and is not particular to FAIMS [49]. These results show the utility of slit ESI/FAIMS interface at liquid flows at least no lower than the maximum $Q=20-40 \mu \mathrm{L} / \mathrm{min}$ found using the single-aperture design at $50{ }^{\circ} \mathrm{C}$ [40]. (Satisfactory performance up to $500 \mu \mathrm{L} / \mathrm{min}$ was reported when the outer electrode comprising the ESI interface was at $90{ }^{\circ} \mathrm{C}$ [7]. Slit ESI/FAIMS interfaces have to be similarly heated for comparison with those results.) While opening the front FAIMS aperture likely facilitates penetration of ESI solvent vapors into the gap, a lower vapor concentration at equal $Q$ (due to vapor production spread over greater volume in multiemitter arrays) offsets that effect at least in part.

\section{Conclusions}

We have developed a planar FAIMS design with entrance and exit (front and back) interfaces featuring slit orifices shaped as a contiguous opening or string of circular apertures. The present device can handle ribbon-shaped ion beams with widths up to $10 \mathrm{~mm}$ from a source to a subsequent stage, such as a MS or drift tube IMS. The slit front interface effectively captures the larger ion currents generated by ESI emitter arrays comprising up to 19 emitters spread over $10 \mathrm{~mm}$, while a slit back interface transmits up to $70 \%$ of filtered ions to the next stage. Extension to larger arrays also seems practical. Compared with conventional ESI/FAIMS/MS platforms with single ESI emitter and circular apertures at the FAIMS entrance and exit, the ion utilization improves by over an order of magnitude. For example, the analyte ion current delivered to the first MS stage 


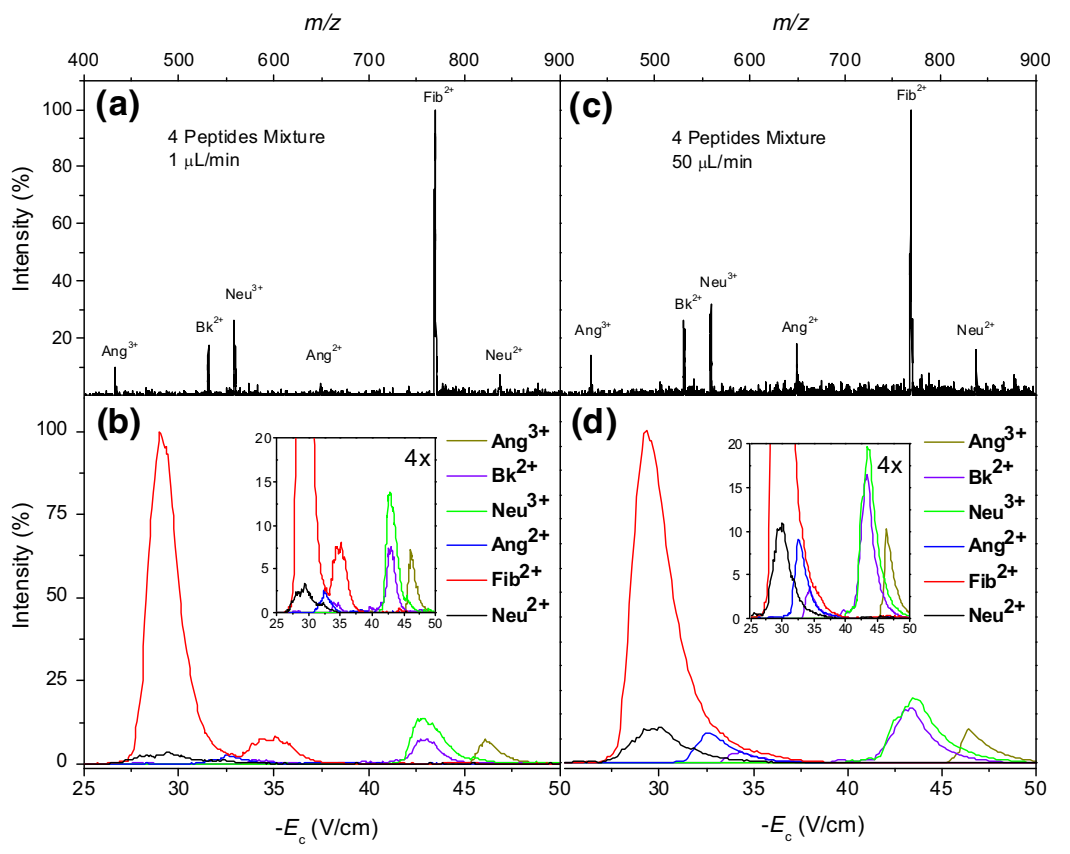

Figure 6. MS (a), (c) and mass-selected FAIMS (b), (d) spectra for peptides measured at ESI liquid flow rates of $1 \mu \mathrm{L} / \mathrm{min}(\mathbf{a}),(\mathbf{b})$ and $50 \mu \mathrm{L} / \min (\mathbf{c})$, (d).

increases from $\sim 6 \mathrm{pA}$ in previous systems to $\sim 250 \mathrm{pA}$. This gain is achieved without degrading FAIMS resolution. The enlarged front interface does not appear to cause a problematic penetration of ESI solvent vapors into the FAIMS gap, allowing robust operation with ESI liquid flow rates up to at least $50 \mu \mathrm{L} / \mathrm{min}$. This work makes planar FAIMS, with its substantial advantages over the cylindrical geometries in terms of resolution, uniform separation power, quantification accuracy, and duty cycle, more attractive for FAIMS /MS and FAIMS / IMS/MS analyses.

\section{Acknowledgments}

The authors thank Dr. Jason S. Page and Bill Danielson for major help with hardware and software development, Dr. Yehia M. Ibrahim for useful comments, and the National Center for Research Resources for support (RR018522). Portions of this work were performed in the Environmental Molecular Sciences Laboratory, a DOE national scientific user facility located at PNNL. PNNL is a multiprogram national laboratory operated by Battelle for the DOE under contract DE-AC05-76RLO 1830.

\section{References}

1. Buryakov, I. A.; Krylov, E. V.; Nazarov, E. G.; Rasulev, U. K. A new method of separation of multi-atomic ions by mobility at atmosphericpressure using a high-frequency amplitude-asymmetric strong electricfield. Int. J. Mass Spectrom. Ion Process 1993, 128, 143-148.

2. Guevremont, R.; Purves, R. W. Atmospheric pressure ion focusing in a high-field asymmetric waveform ion mobility spectrometer. Rev. Sci. Instrum. 1999, 70, 1370-1383.

3. Guevremont, R.; Purves, R. W.; Barnett, D. A.; Ding, L. Y. Ion trapping at atmospheric pressure (760 Torr) and room temperature with a high-field asymmetric waveform ion mobility spectrometer. Int. J. Mass Spectrom. 1999, 193, 45-56.

4. Guevremont, R.; Ding, L. Y.; Ells, B.; Barnett, D. A.; Purves, R. W. Atmospheric pressure ion trapping in a tandem FAIMS-FAIMS coupled to a TOFMS: Studies with electrospray generated gramicidin $\mathrm{S}$ ions. J. Am. Soc. Mass Spectrom. 2001, 12, 1320-1330.
5. Guevremont, R. High-field asymmetric waveform ion mobility spectrometry: A new tool for mass spectrometry. J. Chromatogr. A 2004, 1058 3-19.

6. Guevremont, R.; Makowski, B. Using FAIMS to increase selectivity for LC-MS analyses. Am. Lab. 2005, 37, 11-14.

7. Wu, S. T.; Xia, Y. Q.; Jemal, M. High-field asymmetric waveform ion mobility spectrometry coupled with liquid chromatography/electrospray ionization tandem mass spectrometry (LC/ESI-FAIMS-MS/MS) multi-component bioanalytical method development, performance evaluation, and demonstration of the constancy of the compensation voltage with change of mobile phase composition or flow rate. Rapid Commun. Mass Spectrom. 2007, 21, 3667-3676.

8. Canterbury, J. D.; Yi, X. H.; Hoopmann, M. R.; MacCoss, M. J. Assessing the dynamic range and peak capacity of nanoflow LC-FAIMS-MS on an ion trap mass spectrometer for proteomics. Anal. Chem. 2008, 80, 6888-6897.

9. Venne, K.; Bonneil, E.; Eng, K.; Thibault, P. Improvement in peptide detection for proteomics analyses using nanoLC-MS and high-field asymmetry waveform ion mobility mass spectrometry. Anal. Chem. 2005, 77, 2176-2186.

10. Tang, K. Q.; Li, F. M.; Shvartsburg, A. A.; Strittmatter, E. F.; Smith, R. D. Two-dimensional gas-phase separations coupled to mass spectrometry for analysis of complex mixtures. Anal. Chem. 2005, 77, 6381-6388.

11. Robinson, E. W.; Garcia, D. E.; Leib, R. D.; Williams, E. R. Enhanced mixture analysis of poly(ethylene glycol) using high-field asymmetric waveform ion mobility spectrometry combined with Fourier transform ion cyclotron resonance mass spectrometry. Anal. Chem. 2006, 78, 2190-2198.

12. Sultan, J.; Gabryelski, W. Structural identification of highly polar nontarget contaminants in drinking water by ESI-FAIMS-Q-TOF-MS. Anal. Chem. 2006, 78, 2905-2917.

13. Robinson, E. W.; Williams, E. R. Multidimensional separations of ubiquitin conformers in the gas phase: Relating ion cross sections to H/D exchange measurements. J. Am. Soc. Mass Spectrom. 2005, 16, 1427-1437.

14. Purves, R. W.; Guevremont, R.; Day, S.; Pipich, C. W.; Matyjaszczyk, M. S. Mass spectrometric characterization of a high-field asymmetric waveform ion mobility spectrometer. Rev. Sci. Instrum. 1998, 69, 40944105.

15. Shvartsburg, A. A.; Tang, K.; Smith, R. D. Optimization of the design and operation of FAIMS analyzers. J. Am. Soc. Mass Spectrom. 2005, 16, 2-12.

16. Shvartsburg, A. A.; Tang, K. Q.; Smith, R. D. Modeling the resolution and sensitivity of FAIMS analyses. J. Am. Soc. Mass Spectrom. 2004, 15, 1487-1498.

17. Shvartsburg, A. A.; Tang, K. Q.; Smith, R. D. FAIMS operation for realistic gas flow profile and asymmetric waveforms including electronic noise and ripple. J. Am. Soc. Mass Spectrom. 2005, 16, 1447-1455.

18. Mason, E. A.; McDaniel, E. W. Transport Properties of Ions in Gases, Wiley: New York, 1988. 
19. Kapron, J. T.; Jemal, M.; Duncan, G.; Kolakowski, B.; Purves, R. Removal of metabolite interference during liquid chromatography/ tandem mass spectrometry using high-field asymmetric waveform ion mobility spectrometry. Rapid Commun. Mass Spectrom. 2005, 19, 19791983.

20. Ells, B.; Froese, K.; Hrudey, S. E.; Purves, R. W.; Guevremont, R.; Barnett, D. A. Detection of microcystins using electrospray ionization high-field asymmetric waveform ion mobility mass spectrometry/mass spectrometry. Rapid Commun. Mass Spectrom. 2000, 14, 1538-1542.

21. Kolakowski, B. M.; Mester, Z. Review of applications of high-field asymmetric waveform ion mobility spectrometry (FAIMS) and differential mobility spectrometry (DMS). Analyst 2007, 132, 842-864.

22. Xia, Y. Q.; Wu, S. T.; Jemal, M. LC-FAIMS-MS/MS for quantification of a peptide in plasma and evaluation of FAIMS global selectivity from plasma components. Anal. Chem. 2008, 80, 7137-7143.

23. Hatsis, P.; Kapron, J. T. A review on the application of high-field asymmetric waveform ion mobility spectrometry (FAIMS) in drug discovery. Rapid Commun. Mass Spectrom. 2008, 22, 735-738.

24. O'Donnell, R. M.; Sun, X. B.; Harrington, P. D. Pharmaceutical applications of ion mobility spectrometry. Trac-Trends Anal. Chem. 2008, 27, 44-53.

25. Purves, R. W.; Barnett, D. A.; Guevremont, R. Separation of protein conformers using electrospray-high field asymmetric waveform ion mobility spectrometry-mass spectrometry. Int. J. Mass Spectrom. 2000, 197, 163-177.

26. Guevremont, R.; Purves, R. Comparison of experimental and calculated peak shapes for three cylindrical geometry FAIMS prototypes of differing electrode diameters. J. Am. Soc. Mass Spectrom. 2005, 16, differing

27. Barnett, D. A.; Belford, M.; Dunyach, J. J.; Purves, R. W. Characterization of a temperature-controlled FAIMS system. J. Am. Soc. Mass Spectrom. 2007, 18, 1653-1663.

28. Shvartsburg, A. A.; Li, F. M.; Tang, K. Q.; Smith, R. D. High-resolution field asymmetric waveform ion mobility spectrometry using new planar geometry analyzers. Anal. Chem. 2006, 78, 3706-3714.

29. Eiceman, G. A.; Tadjikov, B.; Krylov, E.; Nazarov, E. G.; Miller, R. A.; Westbrook, J.; Funk, P. Miniature radio-frequency mobility analyzer as a gas chromatographic detector for oxygen-containing volatile organic compounds, pheromones, and other insect attractants. J. Chromatogr. A 2001, 917, 205-217.

30. Miller, R. A.; Eiceman, G. A.; Nazarov, E. G.; King, A. T. A novel micromachined high-field asymmetric waveform-ion mobility spectrometer. Sensors Actuators B Chem. 2000, 67, 300-306.

31. Kendler, S.; Lambertus, G. R.; Dunietz, B. D.; Coy, S. L.; Nazarov, E. G.; Miller, R. A.; Sacks, R. D. Fragmentation pathways and mechanisms of aromatic compounds in atmospheric pressure studied by GC-DMS and DMS-MS. Int. J. Mass Spectrom. 2007, 263, 137-147.

32. Eiceman, G. A.; Nazarov, E. G.; Miller, R. A.; Krylov, E. V.; Zapata, A. M. Micromachined planar field asymmetric ion mobility spectrometer as a gas chromatographic detector. Analyst 2002, 127, 466-471.

33. Shaffer, S. A.; Prior, D. C.; Anderson, G. A.; Udseth, H. R.; Smith, R. D. An ion funnel interface for improved ion focusing and sensitivity using electrospray ionization mass spectrometry. Anal. Chem. 1998, 70, 41114119 .
34. Page, J. S.; Kelly, R. T.; Tang, K.; Smith, R. D. Ionization and transmission efficiency in an electrospray ionization-mass spectrometry interface. J. Am. Soc. Mass Spectrom. 2007, 18, 1582-1590.

35. Tang, K. Q.; Lin, Y. H.; Matson, D. W.; Kim, T.; Smith, R. D. Generation of multiple electrosprays using microfabricated emitter arrays for improved mass spectrometric sensitivity. Anal. Chem. 2001, 73, 1658-1663.

36. Kelly, R. T.; Page, J. S.; Tang, K. Q.; Smith, R. D. Array of chemically etched fused-silica emitters for improving the sensitivity and quantitation of electrospray ionization mass spectrometry. Anal. Chem. 2007, 79, 4192-4198.

37. Kelly, R. T.; Page, J. S.; Marginean, I.; Tang, K. Q.; Smith, R. D. Nanoelectrospray emitter arrays providing inter-emitter electric field uniformity. Anal. Chem. 2008, 80, 5660-5665.

38. Kelly, R. T.; Page, J. S.; Zhao, R.; Qian, W. J.; Mottaz, H. M.; Tang, K.; Smith, R. D. Capillary-based multi nanoelectrospray emitters: Improvements in ion transmission efficiency and implementation with capillary reversed-phase LC-ESI-MS. Anal. Chem. 2008, 80, 143-149.

39. Kim, T.; Tang, K. Q.; Udseth, H. R.; Smith, R. D. A multicapillary inlet jet disruption electrodynamic ion funnel interface for improved sensitivity using atmospheric pressure ion sources. Anal. Chem. 2001, 73, 4162-4170.

40. Kolakowski, B. M.; McCooeye, M. A.; Mester, Z. Compensation voltage shifting in high-field asymmetric waveform ion mobility spectrometrymass spectrometry. Rapid Commun. Mass Spectrom. 2006, 20, 3319-3329.

41. Deng, W. W.; Klemic, J. F.; Li, X. H.; Reed, M. A.; Gomez, A. Increase of electrospray throughput using multiplexed microfabricated sources for the scalable generation of monodisperse droplets. J. Aerosol. Sci. 2006, 37, 696-714.

42. Kelly, R. T.; Page, J. S.; Luo, Q. Z.; Moore, R. J.; Orton, D. J.; Tang, K. Q.; Smith, R. D. Chemically etched open tubular and monolithic emitters for nanoelectrospray ionization mass spectrometry. Anal. Chem. 2006, $78,7796-7801$.

43. Shvartsburg, A. A.; Li, F. M.; Tang, K. Q.; Smith, R. D. Distortion of ion structures by field asymmetric waveform ion mobility spectrometry. Anal. Chem. 2007, 79, 1523-1528.

44. Ibrahim, Y.; Tang, K. Q.; Tolmachev, A. V.; Shvartsburg, A. A.; Smith, R. D. Improving mass spectrometer sensitivity using a high-pressure electrodynamic ion funnel interface. J. Am. Soc. Mass Spectrom. 2006, 17, 1299-1305.

45. Delamora, J. F.; Loscertales, I. G. The current emitted by highly conducting Taylor cones. J. Fluid. Mech. 1994, 260, 155-184.

46. Bocanegra, R.; Galan, D.; Marquez, M.; Loscertales, I. G.; Barrero, A Multiple electrosprays emitted from an array of holes. J. Aerosol. Sci. 2005, 36, 1387-1399.

47. Wilm, M.; Mann, M. Analytical properties of the nanoelectrospray ion source. Anal. Chem. 1996, 68, 1-8.

48. Marginean, I.; Kelly, R. T.; Prior, D. C.; LaMarche, B. L.; Tang, K.; Smith, R. D. Analytical characterization of the electrospray ion source in the nanoflow regime. Anal. Chem. 2008, 80, 6573-6579.

49. Tang, K. Q.; Page, J. S.; Smith, R. D. Charge competition and the linear dynamic range of detection in electrospray ionization mass spectrometry. J. Am. Soc. Mass Spectrom. 2004, 15, 1416-1423. 